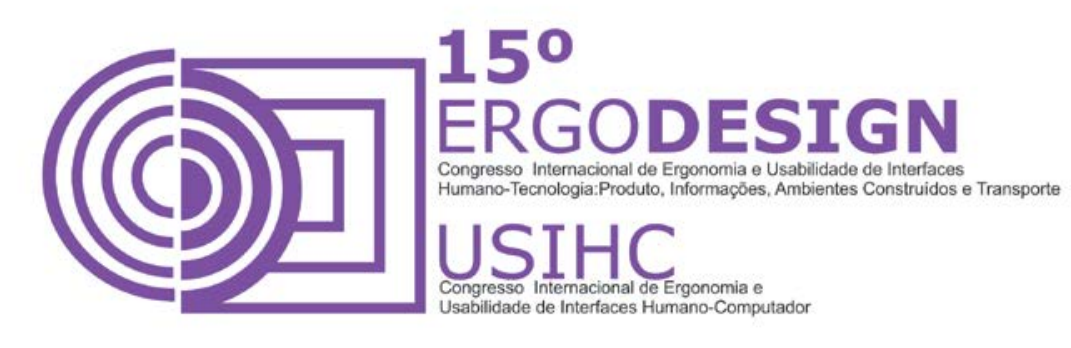

\title{
ERGONOMÍA COMO MEDIO DE TRANSFORMACIÓN ESPACIAL: EL USUARIO Y SU RELACIÓN CON EL LUGAR.
}

\section{ERGONOMICS AS TRANSFORMATION THROUGH SPACE: USER AND ITS RELATIONSHIP WITH PLACE.}

\author{
GALLEGO CARDONA, Héctor Autor (1); \\ Torres Gómez, Ana María (2); \\ Giraldo Vásquez, Natalia (3) \\ (1) LATworkshop, Arquitecto. \\ E-mail: hectorgallego8906@gmail.com \\ (2) LATworkshop, Arquitecta. \\ E-mail:anamariatg28@gmail.com \\ (3) Universidad De San Buenaventura-Medellín, Arquitecta \\ E-mail: ngiladv@gmail.com
}

\begin{abstract}
RESUMEN
Existen individuos que trascienden los criterios estandarizados comunes en una población, bien sea por sus características físicas o mentales. El análisis de estos factores resulta determinante en el inicio de un estudio ergonómico. Este trabajo tiene como objetivo develar el proceso metodológico mediante el cual se generaron estrategias proyectuales para mejorar las condiciones físico-ambientales en el interior de un espacio determinado y para un usuario especifico. Dicho proceso permitió evidenciar la transformación física de un espacio por medio de la implementación de ergonomía, como catalizador de cambios físicos y ambientales.
\end{abstract}

Palabras clave: Arquitectura, Ergonomía, Antropometría, transformación espacial, vivienda. 


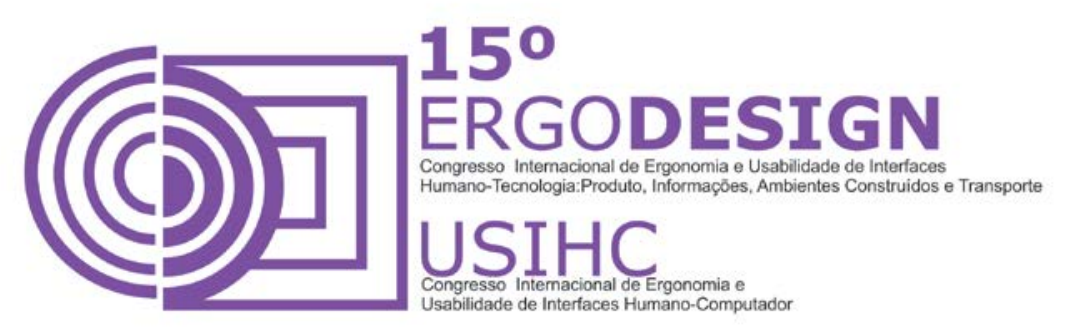

\begin{abstract}
There are individuals that transcend the common standardized criteria in a population, either by physical or mental characteristics. The analysis of these factors is critical for the onset of an ergonomic study. This study aims to reveal the methodological process by which generated design strategies to improve physical and environmental within a given space conditions for a specific user. This process enables the confirmation of transformation from one place through the implementation of ergonomics, as a catalyst for physical changes and environmental.
\end{abstract}

Keywords: Architecture, Ergonomics, Anthropometry, spatial transformation, housing.

\title{
1. INTRODUCCIÓN
}

El presente trabajo se desarrollo dentro del marco académico, con la finalidad de transformar un espacio común (estudio de lectura) diseñado de forma estandarizada el cual en ninguno de sus aspectos garantizaba la calidad en la habitabilidad del lugar (Cardona, Ardila y Castaño 2001). En relación al usuario, actividad desarrollada y espacio. Así fueron implementadas estrategias proyectuales que garantizaran condiciones físicas y ambientales pertinentes según las necesidades ergonómicas particulares del usuario: joven obeso de 24 años con hábitos de lectura.

\section{METODOLOGÍA}

El desarrollo del ejercicio proyectual a través de la ergonomía, fue abordado en principio desde una etapa de diagnóstico por medio del análisis antropométrico del individuo, sus posturas y formas de apropiación del espacio durante el desarrollo de su actividad de lectura. Seguidamente, se dio comienzo a la siguiente etapa en la cual se propuso un dispositivo que permitiera corregir los problemas de postura detectados en el diagnóstico. Terminada esta etapa, se procedió a determinar los factores físico-ambientales (asoleamiento, ventilación, iluminación natural y acústica) que incidían en las formas de acomodación del usuario. Por último, se proponen estrategias estrategias proyectuales con el fin de resolver dichas problemáticas.

\section{DESARROLLO.}

3.1 USUARIO: ergonomía y gesto corporal. Las diferencias en el habitar.

El diagnóstico de los gestos corporales (posturas) asumidos por el individuo al momento de realizar su actividad de lectura, fue analizado con el objetivo de establecer la variabilidad corpórea y las relaciones existentes entre el cuerpo y los objetos de una persona obesa. De esta manera, fueron desarrollados estudios que permitieron determinar la influencia de factores 


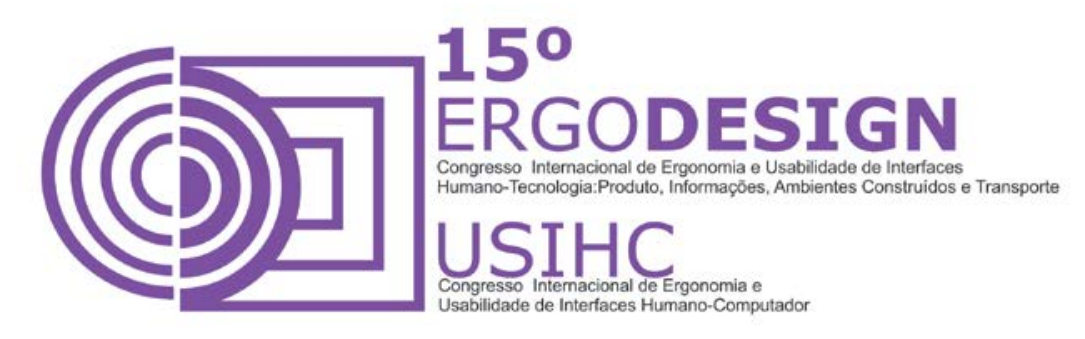

ambientales en las posturas adoptadas por el usuario mientras desarrollaban su actividad. (Ver figura 1).

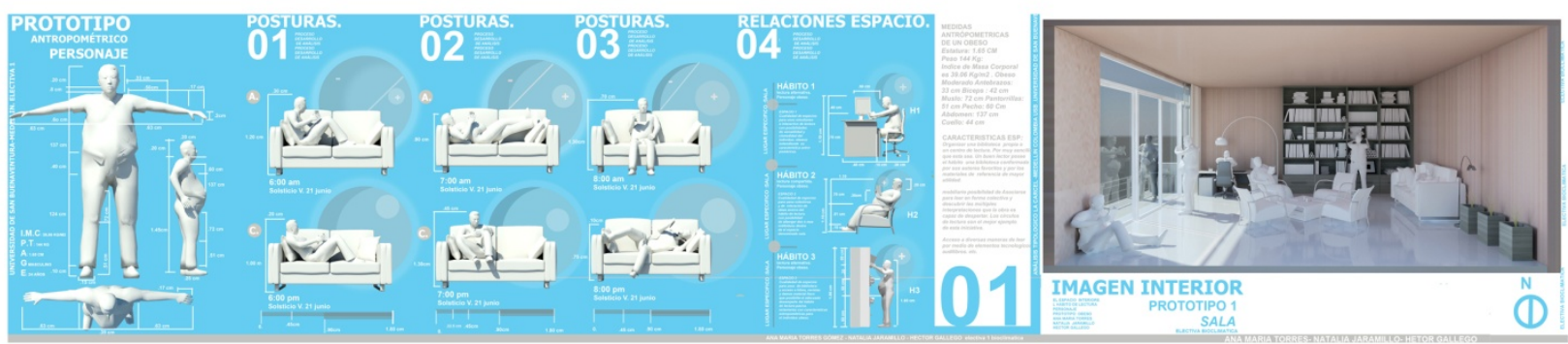

Figura 1. Análisis antropométrico del personaje y gestos corporales

El análisis diagnóstico anterior, expone las posturas del usuario durante la actividad de lectura en las horas de la mañana entre las 6:00am y 8:00am, contrastadas con horas de la noche 6:00 pm y 8:00 pm. Los círculos puestos tras el personaje y el objeto (mueble), revelan la influencia de la cantidad de luz, en las posturas y el ángulo de visualización del libro que adopta el usuario mientras realiza la lectura. Los estudios se llevaron a cabo por medio de métodos experimentales en los cuales el análisis de gestos corporales fue realizado a través de la observación del usuario y fueron registrados en principio por medio de fotografías, las cuales posteriormente se transformaron a modelos tridimensionales por medio del software 3dmax.

\subsection{ERGONOMÌA: Medio de transformación espacial.}

Simultáneamente y tras analizar el impacto de las condiciones físico-ambientales durante el desarrollo de la actividad de lectura, se determinó que, las variables ambientales afectan de manera conjunta la calidad ambiental al interior del espacio y consecutivamente la sensación de confort del usuario mientras ejecuta su actividad. Considerando lo anterior, el diagnóstico realizado: iluminación natural, incursión solar y acústica, aborda un análisis que en conjunto con las estrategias proyectuales y el estudio antropométrico del usuario, permitieron evidenciar la trasformación del espacio físico por medio de la ergonomía como herramienta proyectual para la configuración de espacios habitables. En la figura 2, se observa el desarrollo progresivo del ejercicio.
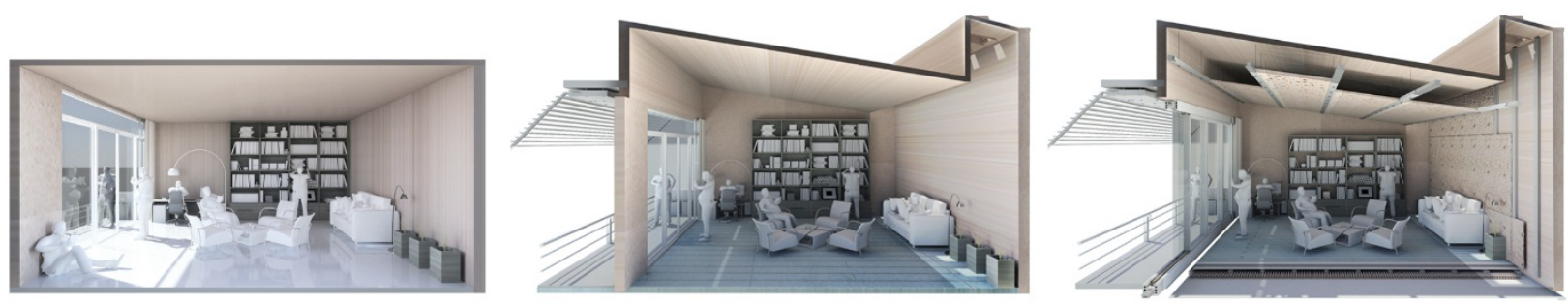


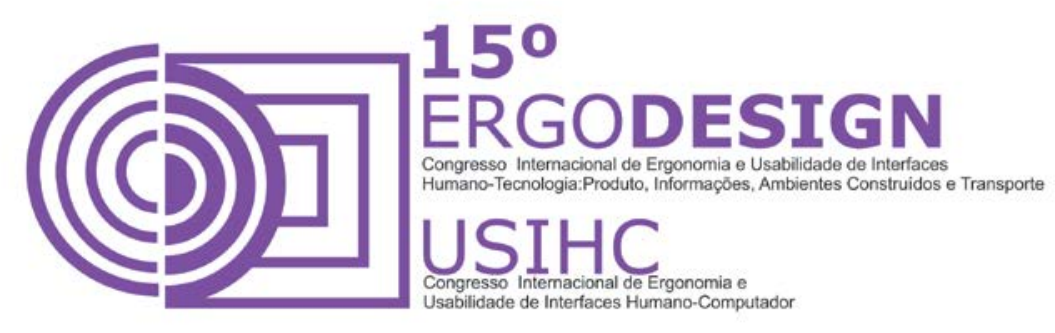

Figura 2. Transformación espacial: iluminación + control solar + acústica.

\section{BIBLIOGRAFÍA}

Cardona, Ader Augusto García, Verónica Henriques Ardila, e Alexander González Castaño. Metodología para la evaluación de puestos de trabajo. Medellín: Grupo EMAT, 2001.

\section{AGRADECIMIENTOS}

El equipo de trabajo agradece a la Arquitecta Natalia Giraldo Vásquez, M.Sc de la Universidad Federal de Santa Catarina - UFSC (Florianópolis, Brasil). Por su acompañamiento durante el proceso de formulación del ejercicio académico y análisis de resultados. 


\section{(C)]}

5. ANEXOS
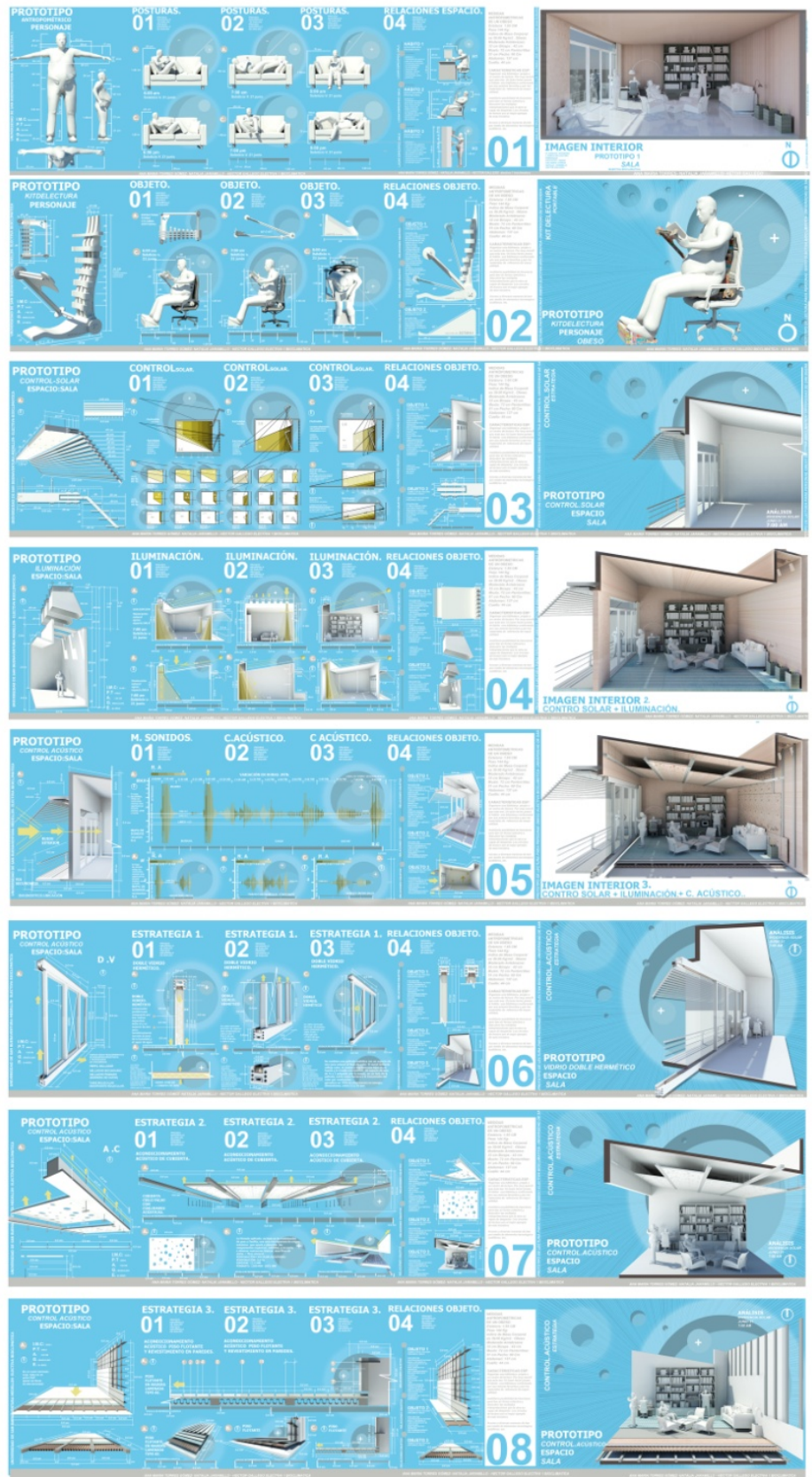

FIGURA 3. Desarrollo del ejercicio proyectual 